\title{
Magneto-ellipsometry as a powerful technique for investigating magneto-optical structures properties
}

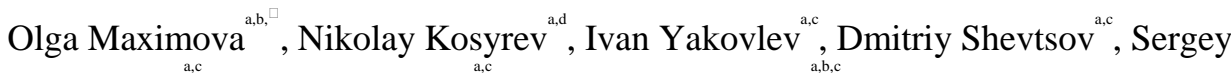 \\ Lyaschenko $^{\text {a.c }}$, Sergey Varnakov ${ }^{\text {a.c }}$, Sergey Ovchinnikov ${ }^{\text {a.be }}$ \\ "Kirensky Institute of Physics, Federal Research Center KSC SB RAS, Krasnoyarsk 660036, Russia ' Siberian Federal \\ University, Krasnoyarsk 660041, Russia 'Reshetnev Siberian State Aerospace University, Krasnoyarsk 660037, Russia ${ }^{d}$ \\ Achinsk Branch of Krasnoyarsk State Agrarian University, Achinsk 662150, Russia
}

\section{ARTICLE INFO ABSTRACT}

Keywords:

Magneto-optical Kerr effect

Ellipsometry In situ

measurements

\begin{abstract}
In this work we report on new magneto-ellipsometry set-up that allows to grow thin films and nanostructures by ultrahigh vacuum thermal evaporation as well as to conduct in situ measurements during the growth in order to analyze and control nanostructures properties. Ellipsometry and transverse magneto-optical Kerr effect measurements can be performed in situ inside this set-up. A uniform magnetic field of high intensity (more than $1 \mathrm{kOe}$ ) can be applied to samples inside the vacuum chamber. Also, we report on the developed method of data interpretation that is the base of the set-up software. Thus, we present a powerful tool for nanostructures synthesis and characterization.
\end{abstract}

\section{Introduction}

It is well-known that new nanomaterial synthesis is needed for various aspects of modern life, as nanostructures are highly appreciated in aerospace technologies, medicine, biology, engineering, civil engineering. That is why the development of non-destructive methods of control and diagnosis is required. For instance, there is a great demand for optical and magneto-optical techniques as they are powerful, give information about magnetic ordering, dominant magnetic anisotropies, correlation of Curie temperature with film thickness, the critical magnetization exponent at the two-dimensional phase transition, spin frustration at the interface between ferromagnetic and antiferromagnetic films, etc [1]. Moreover, these techniques can be easily used for in situ real time control of nanomaterials synthesis, directly in ultrahigh vacuum chamber. One of them is magnetoellipsometry.

There are many papers on magneto-optical ellipsometry, however they mostly present the ex situ investigations [2-6], while our paper offers the solution of the problem of the in situ investigations by means of magneto-ellipsometry.

Carrying out in situ ellipsometric and magneto-ellipsometric analysis involves a number of challenges. There were attempts to design a single setup for determining magneto-optical and conventional optical constants in it. For example, Berger et al. managed to do it, but for that experimental setup it is necessary to measure the reflected light polarization for at least three incoming polarizations [3], so it is quite difficult to be applied for real time in situ process control. Mok et al. [6,7] had to use several setups for complete characterization of the material, for example, he measured magnetization by a superconducting quantum interference device magnetometer and Mueller matrix by the vector-magneto-optical generalized ellipsometer. One more issue is the creation of a combined system of the optical alignment of the sample with simultaneous applying to it a uniform magnetic field of high intensity of more than $1 \mathrm{kOe}$. In the work [8], the authors report about creating a hybrid set-up with helium sample cooling, which is located in high vacuum between the Helmholtz coils with a working field of $400 \mathrm{Oe}$. Nevertheless, this design does not allow to explore the sample without removing it to the air from the processing chamber and the declared value of the magnetic field strength can be clearly not enough.

Besides that, creation of the software for integrated analysis and processing of the parameters that are under study is an important component in the implementation of the in situ magneto-ellipsometry method for the nanomaterials monitoring. It should be applicable to various sample types, so the algorithm should be written for several models of reflective nanostructures. The use of this software should reduce the time of experiment data analysis and increase efficiency.

Thus, we see that we need truly sufficient, simple, and reliable method for spectral in situ magneto-ellipsometric measurements and for interpretation of experimental data. In this paper, we present a new 


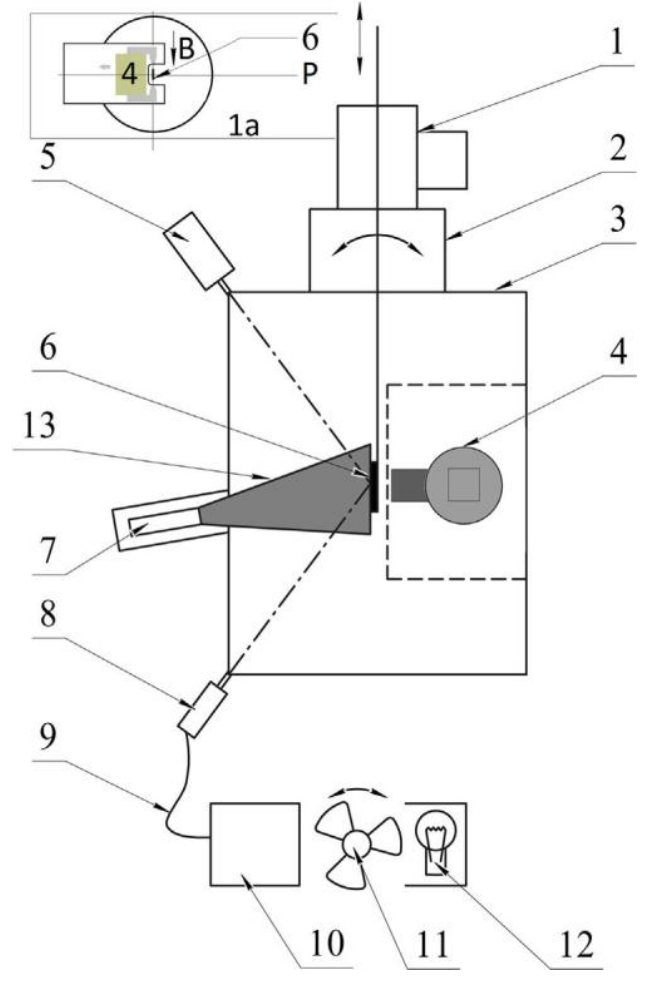

Fig. 1. Schematic diagram of the experimental setup (1 - substrate introduction chamber, 2 - substrate rotation control, 3 - main UHV chamber, 4 - electromagnet $400 \mathrm{mT}, 5$ - analyzer part, 6 - sample, 7 molecular beam, 8 - evaporator, 9 - polarizer part, 10 - optic cable, 11 - spectroscope, 12 - shutter for creation the sync-pulse, 13 - light source; in 1a insert: P is a plane of incidence, B - direction of magnetic field, 4 - electromagnet (topside view) and 6 - sample (topside view)).

in situ setup that consists primarily of a magneto-optical generalized ellipsometer, which is integrated into an ultra-high vacuum chamber with the electromagnet for magnetization reversal of the sample. This setup is used for thin films fabrication by molecular beam epitaxy and for their studies by means of the in situ magneto-optical ellipsometry. Accordingly, we suggest an approach to the interpretation of ellipsometric and magneto-ellipsometric measurements data in order to obtain material parameters of thin films.

\section{Setup and data acquisition}

In order to conduct in situ ellipsometric and magneto-ellipsometric measurements a specialized ultra-high vacuum chamber (UHV) with the possibility of the sample magnetization reversal was created. Its diagram is shown in Fig. 1. This unit is designed for the growth of thin films and nanostructures by UHV thermal evaporation. During a film growth, the polarized light is incident on the substrate 6 and after the reflection the polarization changes are registered by the analyzer part 5 of a high-speed spectroscopic ellipsometer Ellips-1891 [9]. The magnetization reversal of a sample is carried out by a special electromagnet 4 , which is placed outside the vacuum area. The magnet is placed close to the sample using a special port of vacuum chamber (Fig. 1a), which is made so that when the magnet is in operation, the sample is strictly between its poles with the minimum clearance. The sample, being placed inside a vacuum area, is separated by thin walls of stainless steel from the atmosphere, where the electromagnet is located. This construction provides a uniform magnetic field of high intensity.

It should be noted that the magnetic field is perpendicular to the plane of incidence, at the same time it is parallel to the sample plane. Therefore, it is the geometry of transverse magneto-optical Kerr effect.

The electromagnet moves on special rails. It allows to use different magnets according to the task. In addition, to investigate low coercivity samples, these rails allow to move the magnet away to a required distance so that the impact of the remanent magnetization of the electromagnet core is neutralized. Consequently, we can change the magnetic field in a wide range by using only one electromagnet. Thus, the developed complex is designed so that the maximum magnetic field strength is limited only by the electromagnet scheme. A manipulator-holder model includes a lock-chamber that provides gateway loading of investigated structures; a vacuum shutter system that provides the ability of loading without the break of the vacuum in the processing chamber; a vacuum transport system that ensures the installation and alignment of the sample under ellipsometry diagnosis. This system has allowed us to implement the sample holder as a cold finger for measurements in a wide temperature range. Specifications of the designed set-up are spectral range of measurements $350-1000 \mathrm{~nm}$; time scale of ellipsometric spectrum measurement is no more than $20 \mathrm{~s}$; threshold sensitivity of the polarization-optical measurements is no more than 0.5 ang.min

So, we have the UHV chamber and the facility to investigate optical and magnetic properties of nanostructures in situ.

\section{Results and discussion}

In this section we demonstrate the possibilities of our setup and the method. Let us show it on the example of one sample study. The sample was in the form of $\mathrm{Fe}$ layer on the surface of $\mathrm{SiO}_{2} / \mathrm{Si}$ (100). The process of a $\mathrm{SiO} / \mathrm{Si}$ (100) substrate primary chemistry is specified in [10].A polycrystalline Fe film was prepared by ultrahigh vacuum thermal evaporation with deposition on the cool substrate inside the above presented setup. The total time of Fe deposition was $10 \mathrm{~h}$. All the measurements were carried out inside the setup. The $\mathrm{Fe}$ deposition rate was monitored at the light beam wavelength $577 \mathrm{~nm}$. The angle of incidence for in situ measurements was fixed at $56^{\circ}$. The spectral measurements of ellipsometric parameters $\psi$ and $\Delta$ during Fe deposition were held in the four-band regime [11].

In order to determine the structural properties of the Fe layer we performed in situ zero magnetic field ellipsometric measurements within a wavelength range of 400-900 nm. To estimate the Fe film thickness from the in situ spectroscopic ellipsometry measurements data we have carried out modeling of our sample Fe layer ( )/ $\mathrm{SiO}_{2}$ (layer )/ Si substrate

() by the bilayer system. The Nelder- Mead algorithm was used for the thickness optimization [12,13]. The values of $\mathrm{Fe} \mathrm{SiO} \mathrm{,} \mathrm{2,} \mathrm{Si} \mathrm{dielectric}$ permittivity that were necessary for optimization were taken from the experimental works [14-16]. According to the fitting of optical model to sharp interfaces the thickness of the $\mathrm{SiO}_{2}$ layer was $\sim 3.84 \mathrm{~nm}$. The thickness of the Fe was $\sim 17.0 \mathrm{~nm}, \sim 33.0 \mathrm{~nm}, \sim 50.5 \mathrm{~nm}, \sim 65.0 \mathrm{~nm}, \sim 80.25 \mathrm{~nm}$, $\sim 96.0 \mathrm{~nm}, \sim 113.5 \mathrm{~nm}, \sim 128.75 \mathrm{~nm}, \sim 144.5 \mathrm{~nm}, \sim 160.5 \mathrm{~nm}$ after the $1 \mathrm{st}$, the $2 \mathrm{nd}$, the $3 \mathrm{rd}$, the $4 \mathrm{th}$, the $5 \mathrm{th}$, the 6 th, the $7 \mathrm{th}$, the $8 \mathrm{th}$, the 9 th, the $10 \mathrm{th}$ hour of deposition, respectively.

Another thing under in situ control was optical characterization of the sample during deposition. Zero magnetic field ellipsometric measurements were carried out during the process of $\mathrm{Fe} \mathrm{SiO} \mathrm{/} 2 / \mathrm{Si}$ thin film synthesis and resulted in ellipsometric parameters $\psi$ and $\Delta$ that were immediately recalculated into the thickness (d), refractive index

(n) and extinction coefficient (k) of Fe. In Fig. 2 one can see them as a function of the evaporation time. By means of the reported magnetoellipsometry setup the optical properties of the sample can be obtained for a spectral range. We used the developed approach of ellipsometry parameters interpretation and presented in Fig. 3 the calculated values of the refractive index $(\mathrm{n})$ and extinction coefficient $(\mathrm{k})$ of Fe film within a wavelength range of 400-900 nm.

Also, the hysteresis loop can be obtained by means of magnetoellipsometry.

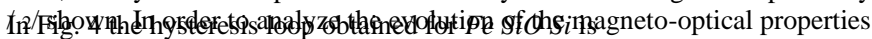
of the sample the in situ measurements of the transverse magneto-optical Kerr effect were carried out during the first $7 \mathrm{~h}$ of $\mathrm{Fe}$ 


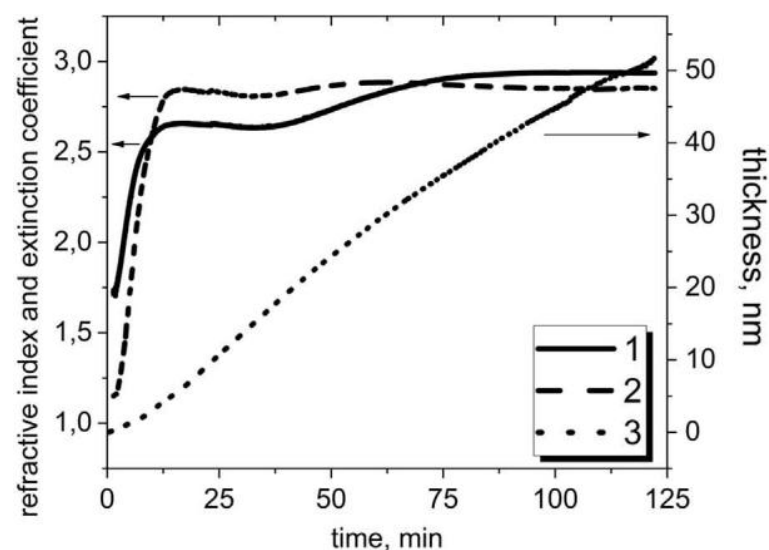

Fig. 2. 1 - extinction coefficient (k), 2 - refractive index (n), 3 - thickness (d) of Fe film as functions of the evaporation time and calculated during the process of $\mathrm{FeS} / \mathrm{iOSithin}$ film growth.

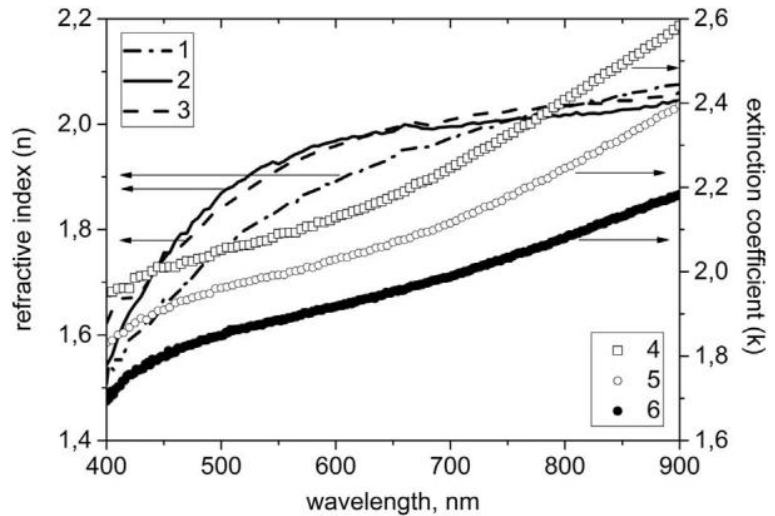

Fig. 3. The spectra of Fe refractive index (n) and Fe extinction coefficient (k), corresponding to different thicknesses (d) of Fe layer during FeS/ iOSithin film gra/ulth: n (d=160.5 nm), $2-\mathrm{n}(\mathrm{d}=36 \mathrm{~nm})$ $3-\mathrm{n}(\mathrm{d}=113.5 \mathrm{~nm}), 4-\mathrm{k}(\mathrm{d}=36 \mathrm{~nm}), 5-\mathrm{k}(\mathrm{d}=113.5 \mathrm{~nm}), 6-\mathrm{k}(\mathrm{d}=160.5 \mathrm{~nm})$.

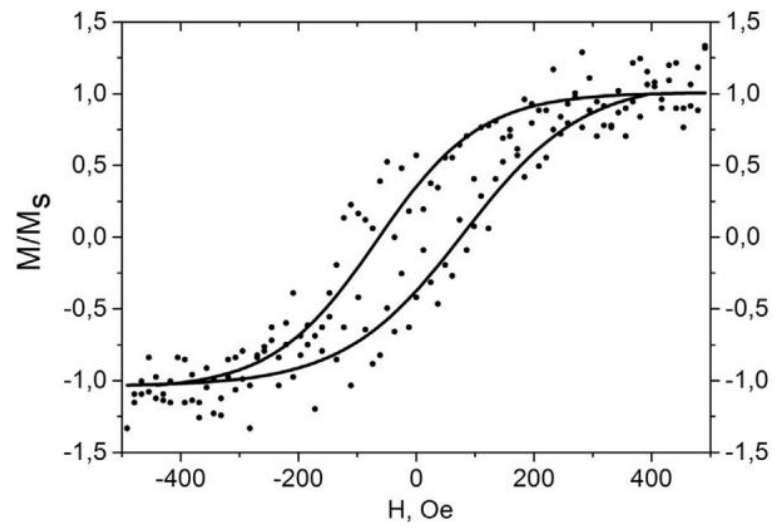

Fig. 4. The hysteresis loop obtained for FeS/ iOSiby means of magneto-ellipsometry.

deposition with a one-hour interval under the magnetization reversal of the sample in the \pm 2 kOe field. In Fig. 5 we demonstrate our results in comparison with some others.

The values of real $\left(Q_{1}\right)$ and imaginary $\left(Q_{2}\right)$ parts of magneto-optical coupling parameter $Q=Q_{1}-i Q_{2}$, as well as the values of refractive index (n) and extinction coefficient $(\mathrm{k})$ of the ferromagnetic metal, have been obtained from the deduced expressions for the bilayer model with the use of the ellipsometric $\left(\psi_{0}\right.$ and $\left.\delta_{0}\right)$ and magneto-ellipsometric $\left(\psi_{0}+\delta \psi\right.$ and $\left.\Delta 0+\delta \Delta\right)$ measurements data. It means that carrying out the measurement of four independent real-valued quantities

$\psi \delta \psi \Delta \delta \Delta)$ sufficient for deriving four real-valued quantities $(,, 0$, is

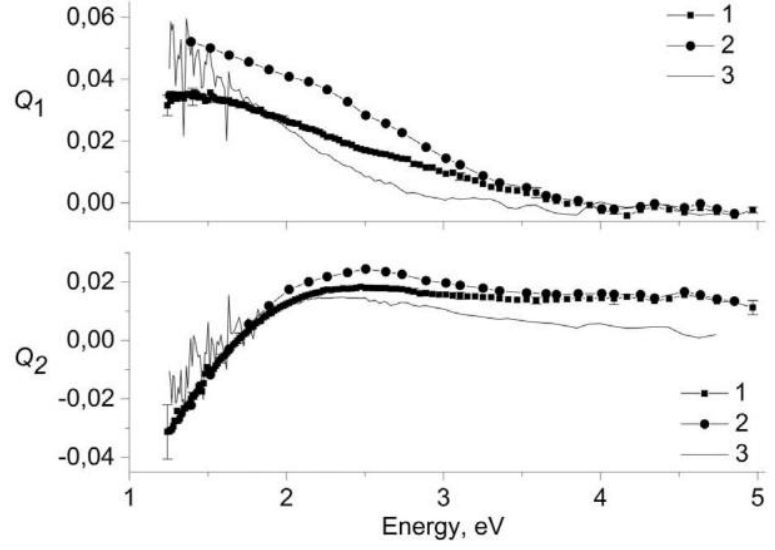

Fig. 5. The values of real $Q 1$ and imaginary ( $Q$ parts of magneto-optical parameter $\mathrm{Q}$,

()2) calculated by various methods: 1) our algorithm that includes Nelder-Mead minimization of the ellipsometric angles using Fe refractive index (n) and extinction coefficient $(\mathrm{k})$ obtained from the ellipsometric measurements of the sample; 2) our algorithm where the values of Fe refractive index (n) and extinction coefficient $(\mathrm{k})$ are taken not from the measurements but from the paper of Johnson and Christy [14]; 3) the values obtained for our sample by the method presented in the work of Neuber at al. [8].

,1(,nkQQ 2). The idea of the approach has been recently published in [17-19]. It is applicable for the case of electromagnetic wave incidence from non-magnetic dielectric medium onto ferromagnetic metal that is deposited on another layer or a substrate. The magnetization vector is z-axis directed, so that YX plane is a plane of incidence, YZ plane is a boundary plane. We consider each interface as each of them impacts the values of ellipsometric angles. The approach is based on the reflection coefficients analysis. Firstly, the basic equation of ellipsometry $[19,20]$ should be written in the following form:

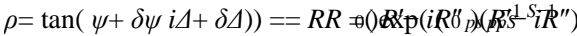

where $\rho$ is the complex ellipsometric parameter, $\mathrm{R}_{\mathrm{p}}$ and $\mathrm{R}_{\mathrm{s}}$ are complex reflection coefficients corresponding to in-plane p-polarization and out-of-plane s-polarization respectively, real parts again are marked by', imaginary by". Secondly, the magnetic field contribution should be distinguished and marked by subscript 1 , non-magnetic summands by subscript 0:

$\left.R_{p}=R_{p p}+R_{p s} R_{p 0 \ddagger^{\prime}}^{\prime} R R i \mathrm{f}^{\prime \prime \prime}\right)_{0} R_{p 1}(2)$

$R_{s}=R_{s s}+R_{s p}=R_{s 0}=R^{\prime}{ }^{s 0}-i R^{\prime \prime} s 0(3)$

One can see that transverse Kerr effect yields to $R^{\prime \prime}=0, R^{\prime}=0 . s 1 s 1$ Then, by substituting Eqs. (2)-(3) into (1) we obtain for non-magnetic condition:

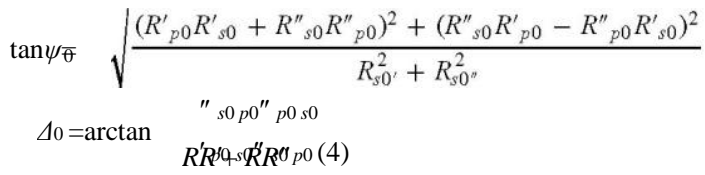

while the influence of an external magnetic field leads to ellipsometric parameters $\delta \Delta a n d \delta$ :

$\delta \Delta \Delta \Delta=-0=\arctan -\Delta_{0}^{R} R_{s \varphi}\left(+^{\prime \prime} 0 R_{P} R^{2}-s^{\prime} \omega f\left(f^{\prime \prime} p\right) R_{1}\right.$

$\delta \psi \psi \psi=-{ }_{0}=\arctan \left(F \tan \left(\psi_{0}\right)\right)-\psi_{0}(5)$

where $\mathrm{F}$ is a helpful notation:

$\tan (\psi$ 他 $\delta \psi)=F \tan \psi$

$F=1+\sqrt{ } 1+\Theta+\Lambda \Theta \Lambda(6)$

where $\Theta$ and $\Lambda$ is: 


$$
\begin{aligned}
& \underline{(R R) R\left(R^{\prime \prime}\right)\left(+2 \operatorname{li}^{2} R^{2} 1 p 1 s 0 p 0 p 1 s 0^{\prime} s 0^{\prime \prime}\right.} \underline{R R R)} \\
& \left(R R^{\prime} \boldsymbol{R} \boldsymbol{R}^{\prime \prime}-\right)^{\prime \prime}+\left({ }^{\prime \prime}{ }^{\prime} R R^{\prime}\right){ }^{2} \\
& (R R+R R)\left(+z^{\prime} R \quad \underline{R R R}\right.
\end{aligned}
$$

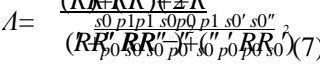

Thus, we presented the key formulae for our data interpretation approach, more details can be found in [17-19].

\section{Conclusions and outlook}

In conclusion, we have presented a new powerful experimental tool for nanomaterials properties study. The setup includes the spectral magneto-ellipsometer that has been set in the UHV growth chamber for in situ diagnostics in real time. The static scheme of the ellipsometric measurements with great potential for the magneto-optical investigations has been chosen The details of the setup have been discussed. The algorithm for the interpretation of magneto-ellipsometric measurement data and the determination of the complex index of refraction and the complex magneto-optical coupling parameter has been proposed. Finally, a complete optical and magneto-optical in situ characterization of the $\mathrm{Fe} / \mathrm{SiO}_{2} / \mathrm{Sistructure}$ has been carried out in order to demonstrate the applications of the tool.

\section{Acknowledgments}

The reported study was funded by Russian Foundation for Basic Research, Government of Krasnoyarsk Territory, Krasnoyarsk Region
Science and Technology Support Fund to the research project No 1642-243058. The work was supported by the Russian Foundation for Basic Research, Grant No. 16-32-00209mol_a, Grant No. 14-0201211, The Complex program of SB RAS No II.2P, project 03582015-0004, the Ministry of Education and Science of the RF (State task No. 16.663.2014K), grant Scientific School 7559.2016.2.

\section{References}

[1] S.D. Bader, J. Magn. Magn. Mater. 100 (1991) 440

[2] P.Q.J. Nederpel, J.W.D. Martens, Rev. Sci. Instrum. 56 (1985) 687

[3] A. Berger, M.R. Pufall, Appl. Phys. Lett. 71 (1997) 965.

[4] G. Neuber, et al., Appl. Phys. Lett. 83 (2003) 4509.

[5] K. Mok, et al., Rev. Sci. Instrum. 82 (2011) 033112

[6] K. Mok, et al., J. Appl. Phys. 110 (2011) 123110.

[7] K. Mok, et al., Phys. Rev. B 84 (2011) 094413.

[8] G. Neuber, et al., Rev. Sci. Instrum. 76 (2005) 023910

[9] V.A. Shvets, et al., Nanotechnol. Russ. 4 (2009) 201. [10] N.V. Volkov, et al., J. Appl. Phys. 109 (2011) 123924

[11] V.A. Shvets, et al., Opt. Spectrosc. 97 (2004) 483.

[12] I.A. Tarasov, et al., Tech. Phys. 57 (2012) 1225.

[13] J.A. Nelder, A.D. Mead, Comput. J. 7 (1965) 308.

[14] P.B. Johnson, R.W. Christy, Phys. Rev. 9 (1974) 5056

[15] I.H. Malitson, J. Opt. Soc. Am. 55 (1965) 1205.

[16] D.E. Aspnes, A.A. Studna, Phys. Rev. B 27 (1983) 985

[17] O.A. Maximova, et al., J. Struct. Chem. 55 (6) (2014) 1134-1141.

[18] O.A. Maximova et al., in: Proceedings of the JEMS-2016, IOP, Glasgow, 2016, pp 117-118.

[19] O.A. Maximova, et al., Vestnik SibGAU 49 (3) (2013) 212-217.

[20] R.M.A. Azzam, N.M. Bashara, Ellipsometry and Polarized Light, North Holland Publishing Company, New York, 1977. 